\title{
An Inverse Scattering Method for a Stratified Slab Using Time-Reversed Fields
}

\author{
Toshifumi Moriyama and Takashi Takenaka \\ School of Engineering \\ Nagasaki University \\ 1-14 Bunkyo-machi, Nagasaki 852-8521, Japan \\ takenaka@nagasaki-u.ac.jp
}

\begin{abstract}
A time-reversal approach is developed for reconstructing electrical parameters of a stratified slab from time -domain reflection and transmission data without using explicitly information on the incident field. The equivalent sources corresponding to the measured reflected and transmitted fields are time-revered. Numerical simulations are performed to reconstruct an image of a stratified dielectric slab.
\end{abstract}

\section{INTRODUCTION}

A significant number of electromagnetic imaging methods have been proposed last decades because of their theoretical interests and practical importance. There are two classes of imaging problems; reconstruction of electrical parameter distributions of scattering objects and detection of targets. The time-reversal (TR) technique was first proposed for acoustic waves and later has also been applied to electromagnetic waves. The main application of TR techniques is detection and identification of point-like targets. There are few papers that deal with reconstruction problem with TR techniques.

In this paper, a time-reversal approach for reconstructing of electrical parameters of a stratified medium from reflection and transmission data without using explicitly information on the incident field is examined. In order to assess the effectiveness of the approach, some numerical simulations are carried out.

\section{FORMULATION}

\section{A. Direct problem}

Let us consider a plane wave normally incident on a stratified slab whose material properties depend only on the $z$ direction, as shown in Figure 1. For simplicity, the material is assumed to be lossless and nondispersive. The plane wave polarized in the $x$-direction is generated by a current source $J_{x}(z, t)=0$ flowing along the $x$-direction on the $z=z_{s}$ plane:

$$
J_{x}(z, t)=I(t) \delta\left(z-z_{s}\right)
$$

where $\delta(t)$ is the Dirac delta function and the time factor $I(t)$ is assumed to be zero before time $t=0$. Then, Maxell's equations can be reduced to

$$
\left[\bar{C} \frac{\partial}{\partial z}-\bar{F} \frac{\partial}{\partial t}\right] \mathbf{v}=\mathbf{j}
$$

where

$$
\begin{array}{llr}
\mathbf{v}=\left(\begin{array}{c}
E_{x}(z, t) \\
H_{y}(z, t)
\end{array}\right) & \text { (3a), } & \mathbf{j}=\left(\begin{array}{c}
J_{x}(z, t) \\
0
\end{array}\right) \\
C=\left(\begin{array}{cc}
0 & -1 \\
-1 & 0
\end{array}\right) & \text { (3c), } & F=\left(\begin{array}{cc}
\varepsilon(z) & 0 \\
0 & \mu(z)
\end{array}\right)
\end{array}
$$

and where $\varepsilon$ and $\mu$ are permittivity and permeability of the medium, respectively. Electromagnetic fields after time $t=0$ are the solutions of Eq. (2) under the initial condition of zero fields $(\mathbf{v}(z, t)=0, t<0)$.

\section{B. Time-reversed field}

The reflected and transmitted fields are measured at the receiver points $z=z_{1}, z_{2}$ during a time interval $[0, T]$. The measured field data is denoted by $\mathbf{v}\left(z_{i}, t\right), i=1,2$. Let us consider the time-reversed fields propagating back into the region $D=\left\{z \mid z_{1}<z<z_{2}\right\}$. The time-reversed fields $\mathbf{u}(z, t)$ is a solution of the following equation:

$$
\left[\bar{C} \frac{\partial}{\partial z}-\bar{F} \frac{\partial}{\partial t}\right] \mathbf{u}=\mathbf{s}_{1}+\mathbf{s}_{2}
$$

where

$$
\mathbf{u}=\left(\begin{array}{l}
u_{1}(z, t) \\
u_{2}(z, t)
\end{array}\right)=\left(\begin{array}{c}
E_{t r}(z, t) \\
H_{t r}(z, t)
\end{array}\right)
$$

under the final condition $\mathbf{u}(z, t)=0(T<t)$. The sources $\mathbf{s}_{1}$ and $\mathbf{s}_{2}$ are the equivalent surface currents $(\mathbf{J}=\mathbf{n} \times \mathbf{H}, \quad \mathbf{M}=\mathbf{E} \times \mathbf{n})$ corresponding to the tangential components of the measured fields $\mathbf{v}\left(z_{i}, t\right)$ at the receiver points $z_{i}, i=1,2$ :

$$
\mathbf{s}_{1}=\left(\begin{array}{c}
-H_{y}(z, t) \\
-E_{x}(z, t)
\end{array}\right) \delta\left(z-z_{1}\right), \mathbf{s}_{2}=\left(\begin{array}{c}
H_{y}(z, t) \\
E_{x}(z, t)
\end{array}\right) \delta\left(z-z_{2}\right)
$$

where the normal vectors $\mathbf{n}$ at $z=z_{1}$ and $z_{2}$ are $\hat{z}$ and $-\hat{z}$, respectively. Note that the forward propagation solution of Eq. (4) under the initial condition $\mathbf{u}(z, t), t<0$ gives an equivalent problem to the original problem of Eq. (2) under the initial condition $\mathbf{v}(z, t), t<0$ in the sense that the forward propagation 
fields of Eq. (4) are the same fields of the original solution of Eq. (2) inside $D$ while it's fields are null outside $D$ [1]. In order to get perfect focusing in a time-reversal process, the necessity of the time-reversed image of an impressed source was pointed out by Carminati et al. [2] and Fink et al. [3]. The timereversed fields are calculated backward in time from $t=T$ to 0 . By using a time variable transformation, a finite difference time domain (FDTD) method can be used to calculate the timereversed fields.

\section{Inverse problem}

The time-domain inverse scattering problem under consideration here is the estimation of $\varepsilon(z)$ and $\mu(z)$ with the knowledge of the measured transient field data $\mathbf{v}\left(z_{i}, t\right), i=1,2$. If the estimated electrical parameters is identical with the true ones, the time-reversed fields produced by the equivalent surface currents $\mathbf{s}_{1}$ and $\mathbf{s}_{2}$ cancel out those by the impressed current $\mathbf{j}$ outside the region $D$ during the time interval $[0, T]$ so that no fields are there. An incorrect estimated electrical parameters, however, does not give null fields outside $D$. Based on this observation, the inverse problem estimating medium parameters is cast as an optimization problem finding a minimizer $\mathbf{p}$ of the following functional:

$$
F(\mathbf{p})=\int_{0}^{T} \int_{\bar{D}}|\mathbf{u}(z, t)|^{2} d z d t
$$

where $\mathbf{p}=(\varepsilon, \mu)$ is the parameter vector, and $\bar{D}$ is the complementary region $\left(-\infty, z_{1}\right] \cup\left[z_{2}, \infty\right)$ of $D$. In numerical simulations, a bounded outside region of $D$ is used as the region $\bar{D}$. If FDTD grid space is restricted to $[0, d]$, then $\bar{D}=\left[0, z_{1}\right] \cup\left[z_{2}, d\right]$.

\section{NUMERICAL RESULTS}

Two numerical simulations are carried out for a stratified dielectric slab of $0.4 \mathrm{~m}$ width by using the FDTD with grid space of 400 cells of cell size $\Delta z=0.01 \mathrm{~m}$ and time step size $\Delta t$ $=30$ psec. The background medium is free space. A source point is located at $z_{\mathrm{s}}=1.5 \mathrm{~m}$ and the time factor $I(t)$ is $\exp \left(-\alpha\left(t-2 t_{0}\right)^{2}\right)$ with $t_{0}=100 \Delta t$ and $\alpha=\left(4 / t_{0}\right)^{2}$. The observation points are located at $z_{1}=0.5 \mathrm{~m}$ and $z_{2}=3.5 \mathrm{~m}$.

The first example is to show numerically the validity of the proposed method. Figure 2a shows the electric field

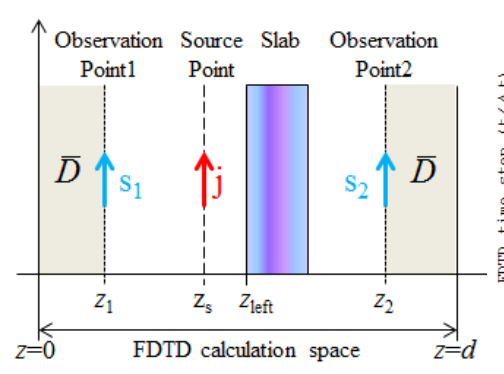

Figure 1. Geometrical configuration of problem. distribution in time-space for a homogeneous slab with $\varepsilon_{r}=4.0$ calculated by Eq. (2), while Fig. $2 \mathrm{~b}$ shows the time-reversed one based on Eq. (4). The electric field distribution with the use of an incorrect permittivity $\left(\varepsilon_{r}=1.5\right)$ in Eq. (4) is shown in Fig. 2c. The leaked fields are observed outside the region $D$.

Next example is reconstruction of a four-layered slab with the relative permittivities 2.0, 3.0, 4.0 and 2.0. A width of each layer is $0.1 \mathrm{~m}$. A genetic algorithm (GA), which is a global optimization technique, is applied to the minimization of the functional (7). The unknown parameters to be estimated are the position of the left face of the slab $z_{\text {left }}$, the permittivities of four layers $\varepsilon_{\mathrm{r} 1}, \varepsilon_{\mathrm{r} 2}, \varepsilon_{\mathrm{r} 3}$ and $\varepsilon_{\mathrm{r} 4}$. The total width $0.4 \mathrm{~m}$ of the slab is assumed to be known. Since the parameter vector $\mathbf{p}$ is a function of $\mathbf{q}=\left(\mathrm{z}_{\mathrm{left}}, \varepsilon_{\mathrm{r} 1}, \varepsilon_{\mathrm{r} 2}, \varepsilon_{\mathrm{r} 3}, \varepsilon_{\mathrm{r} 4}\right)$ specifying the slab, the argument of the functional $F$ given by Eq.(7) is changed from $\mathbf{p}$ to q. Table 1 shows the estimated parameters of the stratified slab after 400 iterations by GA. It is confirmed that the estimated parameters are very accurate.

TABLE I. THE SLAB PARAMETERS ESTIMATED BY GA.

\begin{tabular}{|c|c|c|c|c|c|}
\hline & $z_{\text {left }}[\mathrm{m}]$ & $\varepsilon_{\mathrm{r} 1}$ & $\varepsilon_{\mathrm{r} 2}$ & $\varepsilon_{\mathrm{r} 3}$ & $\varepsilon_{\mathrm{r} 4}$ \\
\hline True & 2.0 & 2.0 & 3.0 & 4.0 & 2.0 \\
\hline GA & 2.0 & 1.8 & 3.1 & 3.9 & 2.1 \\
\hline
\end{tabular}

\section{CONCLUSIONS}

An inverse scattering approach using time-revered fields to reconstruct electrical parameters of a planar stratified media has been proposed. Numerical simulations have demonstrated the validity of our inverse technique. The extension of our approach to multidimensional cases is straightforward.

\section{REFERENCES}

[1] R. F. Harrington, Time-Harmonic Electromagnetic Fields, Wiley-IEEE Press; 2nd edition, 2001.

[2] R. Carminati, J. J. Saenz, J.-J. Greffet, and M. Nieto-Vesperinas, "Reciprosity, unitarity, and time-reversal symmetry of the s matrix of fields containing evanecent components," Phys. Rev. A, vol. 62, 012712 2000

[3] J. de Rosny, and M. Fink, "Overcoming the diffraction limit in wave physics using a time-reversal mirror and a novel acoustic sink," Phys. Rev. Lett. vol. 89. 124301(1)-(4), 2002.

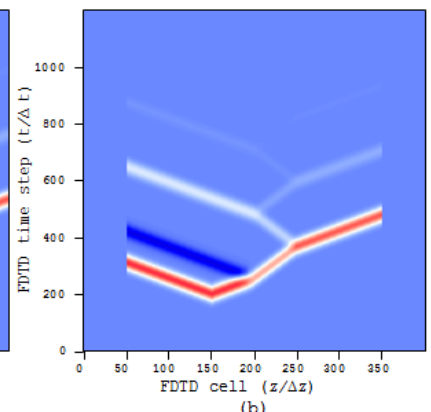

(b)

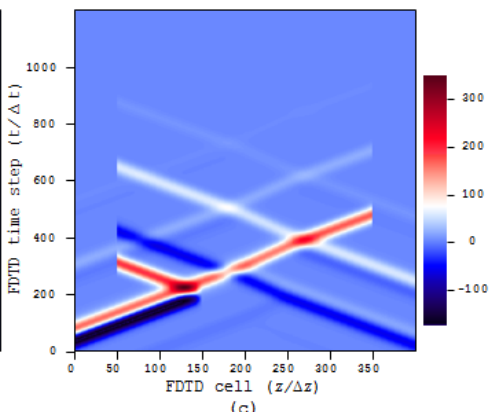

(c)
Figure 2. Electric field distributions in time-space: (a) Forward propagation field, (b) Time-reversed field with true permittivity, (c) Time-reversed field with an incorrect permittivity. 